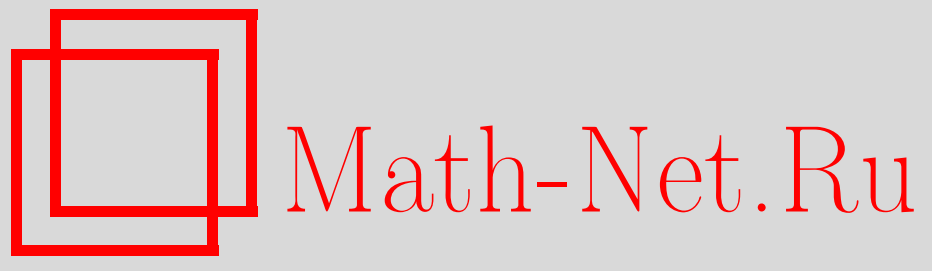

S. E. Graversen, G. Peskir, A. N. Shiryaev, Stopping Brownian motion without anticipation as close as possible to its ultimate maximum, Теория вероятн. и ее примен., 2000, том 45, выпуск $1,125-136$

DOI: https://doi.org/10.4213/tvp327

Использование Общероссийского математического портала Math-Net.Ru подразумевает, что вы прочитали и согласны с пользовательским соглашением http://www . mathnet.ru/rus/agreement

Параметры загрузки:

IP : 35.173 .219 .12

26 апреля 2023 г., 07:52:08

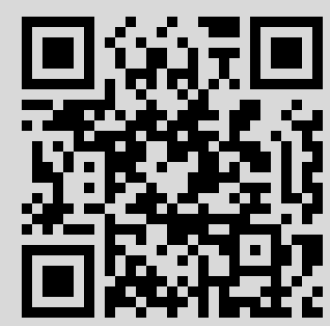


(C) 2000 г. GRAVERSEN S. E.*, PESKIR G.**, SHIRYAEV A. N.** $^{* *}$

\section{STOPPING BROWNIAN MOTION WITHOUT ANTICIPATION AS CLOSE AS POSSIBLE TO ITS ULTIMATE MAXIMUM ${ }^{1)}$}

\section{Dedicated to Yu. V. Prokhorov on his 70th birthday}

Пусть $B=\left(B_{t}\right)_{0 \leqslant t \leqslant 1}-$ стандартное броуновское движение, выходящее из нуля, и пусть $S_{t}=\max _{0 \leqslant r \leqslant t} B_{r}$ для $0 \leqslant t \leqslant 1$. Рассмотрим задачу оптимальной остановки

$$
V_{*}=\inf _{\tau} \mathbf{E}\left(B_{\tau}-S_{1}\right)^{2}
$$

где инфимум берется по всем моментам остановки (относительно $B$ ) таким, что $0 \leqslant \tau \leqslant 1$. Мы показываем, что этот инфимум достигается на моменте остановки $\tau_{*}=\inf \left\{0 \leqslant t \leqslant 1 \mid S_{t}-\right.$ $\left.B_{t} \geqslant z_{*} \sqrt{1-t}\right\}$, где $z_{*}=1.12 \ldots-$ единственный корень уравнения $4 \Phi\left(z_{*}\right)-2 z_{*} \varphi\left(z_{*}\right)-3=0$ с $\varphi(x)=(1 / \sqrt{2 \pi}) e^{-x^{2} / 2}$ и $\Phi(x)=$ $\int_{-\infty}^{x} \varphi(y) d y$. Величина $V_{*}$ равна $2 \Phi\left(z_{*}\right)-1$. Метод доказательства основан на представлении $S_{1}$ в виде стохастического интеграла, случайной замене времени и решении задачи Стефана со свободной границей.

Ключевые слова и фразы: марковский процесс, диффузия, броуновское движение, оптимальная остановка, глобальный максимум, задача Стефана со свободной границей, теорема Ито-Кларка.

1. Formulation of the problem. Imagine the real-line movement of a Brownian particle started at 0 during the time interval $[0,1]$. Let $S_{1}$ denote the maximal height that the particle ever reaches during this time interval. As $S_{1}$ is a random quantity whose values depend on the entire Brownian path over the time interval, its ultimate value is at any given time $t \in[0,1)$

*Institute of Mathematics, University of Aarhus, Ny Munkegade, 8000 Aarhus, Denmark; e-mail: matseg@imf.au.dk

${ }^{* *}$ Institute of Mathematics, University of Aarhus, Ny Munkegade, 8000 Aarhus, Denmark; e-mail: goran@imf.au.dk, www.imf.au.dk/ goran (Department of Mathematics, University of Zagreb, Bijenicka 30, 10000 Zagreb, Croatia).

*** Математический институт им. В. А. Стеклова РАН, ул. Губкина, 8, 117966 Москва, ГСП-1, Россия; e-mail: shiryaev@mi.ras.ru

1) Centre for Mathematical Physics and Stochastics (Aarhus University), supported by the Danish National Research Foundation. 
unknown. Following the Brownian particle from the initial time 0 onward, the question arises naturally as to determine a time when the movement should be terminated so that the position of the particle at that time is as «close» as possible to the ultimate maximum $S_{1}$. In this paper we present the solution to this problem if «closeness» is measured by a mean-square distance.

To formulate the problem more precisely, let $B=\left(B_{t}\right)_{0 \leqslant t \leqslant 1}$ be a standard Brownian motion $\left(B_{0}=0, \mathbf{E} B_{t}=0, \mathbf{E} B_{t}^{2}=t\right)$ defined on a probability space $(\Omega, \mathscr{F}, \mathbf{P})$, and let $\mathbb{F}^{B}=\left(\mathscr{F}_{t}^{B}\right)_{0 \leqslant t \leqslant 1}$ denote the natural filtration generated by $B$. Letting $\mathscr{M}$ denote the family of all stopping (Markov) times $\tau$ with respect to $\mathbb{F}^{B}$ satisfying $0 \leqslant \tau \leqslant 1$, the problem is to compute

$$
V_{*}=\inf _{\tau \in \mathcal{M}} \mathbf{E}\left(B_{\tau}-\max _{0 \leqslant t \leqslant 1} B_{t}\right)^{2}
$$

and to find an optimal stopping time (the one at which the infimum in (1.1) is attained).

The solution to this problem is presented in Theorem 1 below. It turns out that the maximum process $S=\left(S_{t}\right)_{0 \leqslant t \leqslant 1}$ given by

$$
S_{t}=\sup _{0 \leqslant s \leqslant t} B_{s}
$$

and the CUSUM-type reflected process $S-B=\left(S_{t}-B_{t}\right)_{0 \leqslant t \leqslant 1}$ play a key role in the solution.

The optimal stopping problem (1.1) is of interest, for example, in financial mathematics and financial engineering where an optimal decision (i.e., optimal stopping time) should be based on a prediction of the future behaviour of the observable process (asset price, index, etc.). The argument also carries over to many other applied problems where such predictions play a role.

2. The result and proof. The main result of the paper is contained in the following theorem. Below we let

$$
\varphi(x)=\frac{1}{\sqrt{2 \pi}} e^{-x^{2} / 2} \text { and } \Phi(x)=\int_{-\infty}^{x} \varphi(y) d y \quad(x \in \mathbf{R})
$$

denote the density and distribution function of a standard normal variable.

Theorem 1. Consider the optimal stopping problem (1.1) where $\left(B_{t}\right)_{0 \leqslant t \leqslant 1}$ is a standard Brownian motion. Then the value $V_{*}$ is given by the formula

$$
V_{*}=2 \Phi\left(z_{*}\right)-1=0.73 \ldots,
$$

where $z_{*}=1.12 \ldots$ is the unique root of the equation

$$
4 \Phi\left(z_{*}\right)-2 z_{*} \varphi\left(z_{*}\right)-3=0
$$




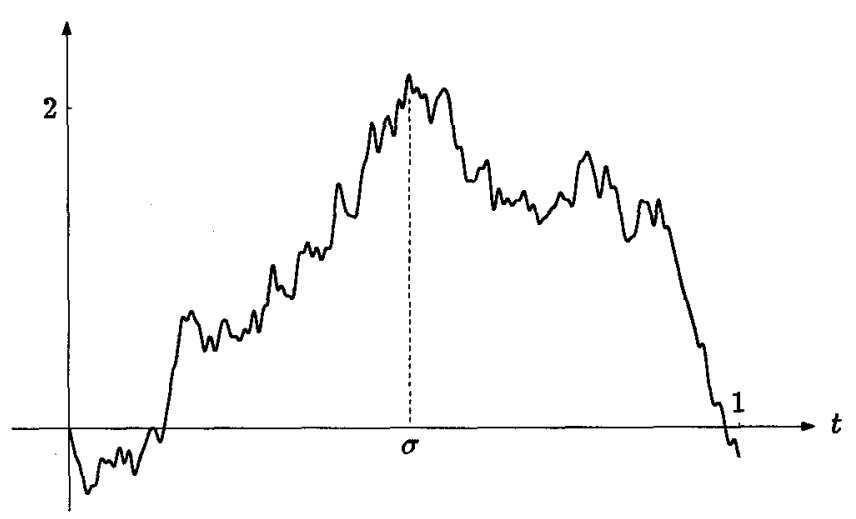

Fig. 1. A computer simulation of a Brownian path $\left(B_{t}(\omega)\right)_{0 \leqslant t \leqslant 1}$ with the maximum being attained at $\sigma=0.51$

and the following stopping time is optimal (see Figures 1-4):

$$
\tau_{*}=\inf \left\{0 \leqslant t \leqslant 1 \mid S_{t}-B_{t} \geqslant z_{*} \sqrt{1-t}\right\}
$$

where $S_{t}$ is given by (1.2) above.

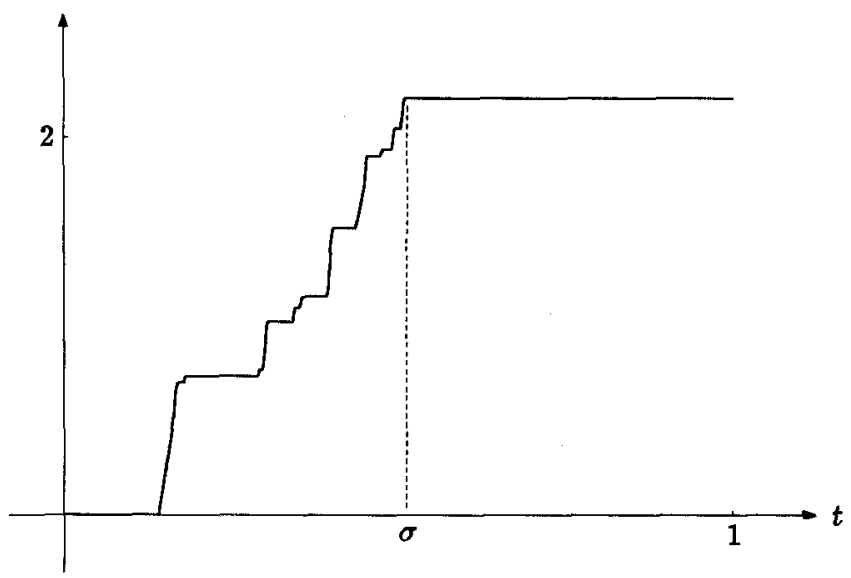

Fig. 2. A computer drawing of the maximum process $\left(S_{t}(\omega)\right)_{0 \leqslant t \leqslant 1}$ associated with the Brownian path from Fig. 1

P r o of. Since $S_{1}=\sup _{0 \leqslant s \leqslant 1} B_{s}$ is a square-integrable functional of the Brownian path on $[0,1]$, by the Itô-Clark representation theorem (see e.g. $\left[2\right.$, p. 191]) there exists a unique $\mathbb{F}^{B}$-adapted process $H=\left(H_{t}\right)_{0 \leqslant t \leqslant 1}$ 


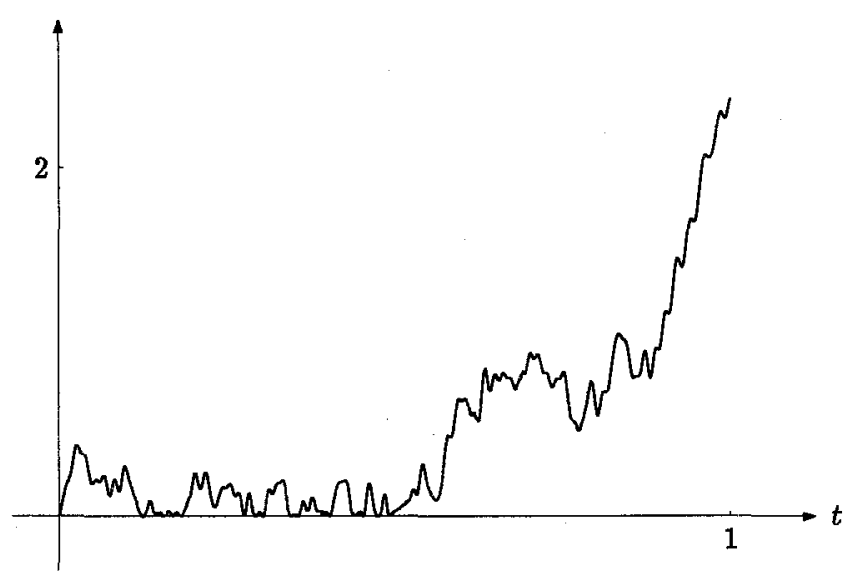

Fig. 3. A computer drawing of the difference process $\left(S_{t}(\omega)-B_{t}(\omega)\right)_{0 \leqslant t \leqslant 1}$ from Figs. 1,2

satisfying $\mathbf{E} \int_{0}^{1} H_{t}^{2} d t<\infty$ such that

$$
S_{1}=a+\int_{0}^{1} H_{t} d B_{t}
$$

where $a=\mathbf{E} S_{1}$. Moreover, the following explicit formula is known to be valid:

$$
H_{t}=2\left(1-\Phi\left(\frac{S_{t}-B_{t}}{\sqrt{1-t}}\right)\right)
$$

for $0 \leqslant t \leqslant 1$ (see e.g. [3, p. 93] and [1, p. 365], or Section 3 below for a direct argument).

1. Associate with $H$ the square-integrable martingale $M=\left(M_{t}\right)_{0 \leqslant t \leqslant 1}$ given by

$$
M_{t}=\int_{0}^{t} H_{s} d B_{s}
$$

By the martingale property of $M$ and the optional sampling theorem, we obtain

$$
\begin{aligned}
\mathbf{E}\left(B_{\tau}-S_{1}\right)^{2} & =\mathbf{E}\left|B_{\tau}\right|^{2}-2 \mathbf{E}\left(B_{\tau} M_{1}\right)+\mathbf{E}\left|S_{1}\right|^{2} \\
& =\mathbf{E} \tau-2 \mathbf{E}\left(B_{\tau} M_{\tau}\right)+1=\mathbf{E}\left(\int_{0}^{\tau}\left(1-2 H_{t}\right) d t\right)+1
\end{aligned}
$$

for all $\tau \in \mathscr{M}$ (recall that $\left.S_{1} \stackrel{\text { law }}{=}\left|B_{1}\right|\right)$. Inserting (2.6) into (2.8) we see that (1.1) can be rewritten as

$$
V_{*}=\inf _{\tau \in \mathscr{M}} \mathbf{E}\left(\int_{0}^{\tau} F\left(\frac{S_{t}-B_{t}}{\sqrt{1-t}}\right) d t\right)+1
$$




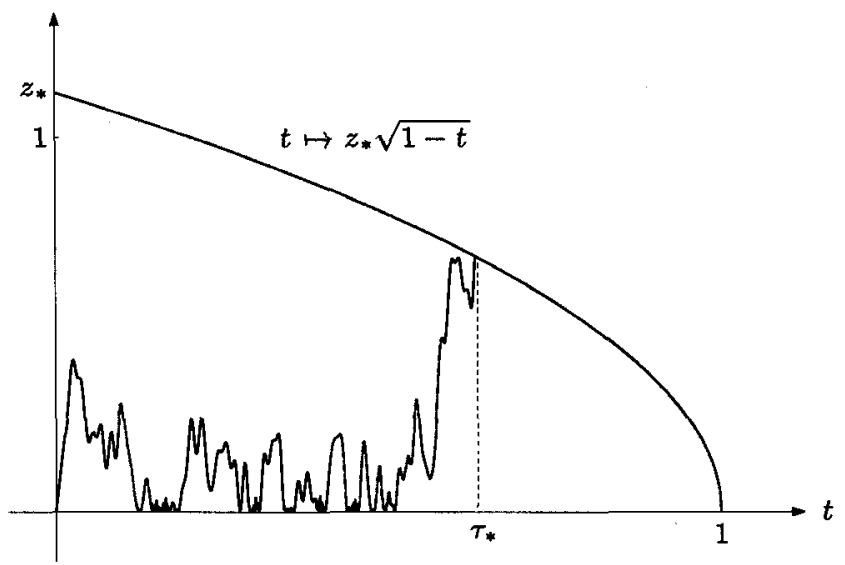

Fig. 4. A computer drawing of the optimal stopping strategy (2.4) for the Brownian path from Figs. 1-3. It turns out that $\tau_{*}=0.62$ in this case (cf. Fig. 1)

where we denote $F(x)=4 \Phi(x)-3$.

Since $S-B=\left(S_{t}-B_{t}\right)_{0 \leqslant t \leqslant 1}$ is a Markov process for which the natural filtration $\mathbb{F}^{S-B}$ coincides with the natural filtration $\mathbb{F}^{B}$, it follows from general theory of optimal stopping (see [4]) that in (2.9) we need only consider stopping times which are hitting times for $S-B$. Recalling moreover that $S-B \stackrel{\text { law }}{=}|B|$ by Lévy's distributional theorem (see e.g. [2, p. 230]) and once more appealing to general theory, we see that $(2.9)$ is equivalent to the optimal stopping problem

$$
V_{*}=\inf _{\tau \in \mathscr{M}} \mathbf{E}\left(\int_{0}^{\tau} F\left(\frac{\left|B_{t}\right|}{\sqrt{1-t}}\right) d t\right)+1 .
$$

In our treatment of this problem, we first make use of a deterministic change of time.

2. Motivated by the form of (2.10), consider the process $Z=\left(Z_{t}\right)_{t \geqslant 0}$ given by

$$
Z_{t}=e^{t} B_{1-e^{-2 t}}
$$

By Itô's formula we find that $Z$ is a (strong) solution of the linear stochastic differential equation

$$
d Z_{t}=Z_{t} d t+\sqrt{2} d \beta_{t}
$$

where $Z_{0}=0$ and the process $\beta=\left(\beta_{t}\right)_{0 \leqslant t \leqslant 1}$ is given by

$$
\beta_{t}=\frac{1}{\sqrt{2}} \int_{0}^{t} e^{s} d B_{1-e^{-2 s}}=\frac{1}{\sqrt{2}} \int_{0}^{1-e^{-2 t}} \frac{1}{\sqrt{1-s}} d B_{s} .
$$

As $\beta$ is a continuous Gaussian martingale with mean zero and variance equal to $t$, it follows by Lévy's characterisation theorem (see e.g. $[2$, p. 142]) that $\beta$ 
is a standard Brownian motion. We thus may conclude that $Z$ is a diffusion process with the infinitesimal generator given by

$$
\mathbb{L}_{Z}=z \frac{d}{d z}+\frac{d^{2}}{d z^{2}}
$$

Substituting $t=1-e^{-2 s}$ in (2.10) and using (2.11), we obtain

$$
V_{*}=2 \inf _{\tau \in \mathscr{M}} \mathbf{E}\left(\int_{0}^{\sigma_{\tau}} e^{-2 s} F\left(\left|Z_{s}\right|\right) d s\right)+1
$$

upon setting $\sigma_{\tau}=\ln (1 / \sqrt{1-\tau})$. It is clear from (2.11) that $\tau$ is a stopping time with respect to $\mathbb{F}^{B}$ if and only if $\sigma_{\tau}$ is a stopping time with respect to $\mathbb{F}^{Z}$. This shows that our initial problem (1.1) reduces to solve

$$
W_{*}=\inf _{\sigma} \mathbf{E}\left(\int_{0}^{\sigma} e^{-2 s} F\left(\left|Z_{s}\right|\right) d s\right)
$$

where the infimum is taken over all $\mathbb{F}^{Z}$-stopping times $\sigma$ with values in $[0, \infty]$. This problem belongs to the general theory of optimal stopping for time-homogeneous Markov processes (see [4]).

3. To calculate (2.16) define

$$
W_{*}(z)=\inf _{\sigma} \mathbf{E}_{z}\left(\int_{0}^{\sigma} e^{-2 s} F\left(\left|Z_{s}\right|\right) d s\right)
$$

for $z \in \mathbf{R}$, where $\mathbf{E}_{z}$ is an averaging with respect to the measure $\mathbf{P}_{z}$ of the process $Z=\left(Z_{t}\right)_{t \geqslant 0}$ with differential (2.12), $Z_{0}=z$, and the infimum is taken as above. General theory combined with basic properties of the map $z \mapsto F(|z|)$ prompts that the stopping time

$$
\sigma_{*}=\inf \left\{t>0:\left|Z_{t}\right| \geqslant z_{*}\right\}
$$

should be optimal in (2.17), where $z_{*}>0$ is a constant to be found.

To determine $z_{*}$ and compute the value function $z \mapsto W_{*}(z)$ in (2.17), it is a matter of routine to formulate the following free-boundary (Stefan) problem:

$$
\begin{array}{rlrl}
\left(\mathbb{L}_{z}-2\right) W(z) & =-F(|z|) \quad \text { for } z \in\left(-z_{*}, z_{*}\right) \\
W\left( \pm z_{*}\right) & =0 & \text { (instantaneous stopping) } \\
W^{\prime}\left( \pm z_{*}\right) & =0 & \text { (smooth fit) }
\end{array}
$$

where $\mathbb{L}_{Z}$ is given by (2.14) above. We shall extend the solution of (2.19)(2.21) by setting its value equal to 0 for $z \notin\left(-z_{*}, z_{*}\right)$, and thus the map so obtained will be $C^{2}$ everywhere on $\mathbf{R}$ but at $-z_{*}$ and $z_{*}$ where it is $C^{1}$. 
Inserting $\mathbb{L}_{Z}$ from (2.14) into (2.19) leads to the following equation:

$$
W^{\prime \prime}(z)+z W^{\prime}(z)-2 W(z)=-F(|z|)
$$

for $z \in\left(-z_{*}, z_{*}\right)$. The form of the equation (2.12) and (2.16) indicate that $z \mapsto W_{*}(z)$ should be even; thus we shall additionally impose

$$
W^{\prime}(0)=0
$$

and consider (2.22) only for $z \in\left[0, z_{*}\right)$.

The general solution of the equation (2.22) for $z \geqslant 0$ is given by

$$
W(z)=C_{1}\left(1+z^{2}\right)+C_{2}\left(z \varphi(z)+\left(1+z^{2}\right) \Phi(z)\right)+2 \Phi(z)-\frac{3}{2} .
$$

The three conditions $W\left(z_{*}\right)=W^{\prime}\left(z_{*}\right)=W^{\prime}(0)=0$ determine constants $C_{1}, C_{2}$ and $z_{*}$ uniquely; it is easily verified that $C_{1}=\Phi\left(z_{*}\right), C_{2}=-1$, and $z_{*}$ is the unique root of the equation (2.3). Inserting this back into (2.22), we obtain the following candidate for the value (2.17):

$$
W(z)=\Phi\left(z_{*}\right)\left(1+z^{2}\right)-z \varphi(z)+\left(1-z^{2}\right) \Phi(z)-\frac{3}{2}
$$

when $z \in\left[0, z_{*}\right]$, upon extending it to an even function on $\mathbf{R}$ as indicated above (see Figure 5).

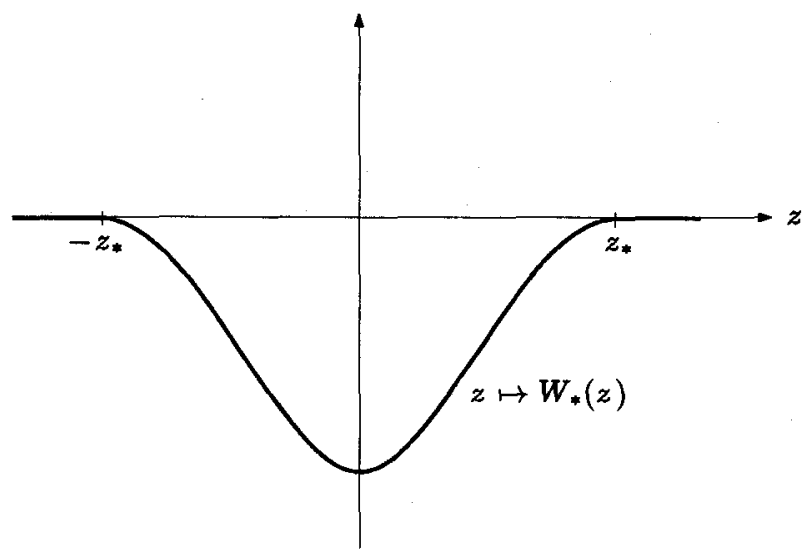

Fig. 5. A computer drawing of the map (2.17). The smooth fit (2.21) holds at $-z_{*}$ and $z_{*}$

To verify that this solution $z \mapsto W(z)$ coincides with the value function (2.17), and that $\sigma_{*}$ from (2.18) is an optimal stopping time, we shall note that $z \mapsto W(z)$ is $C^{2}$ everywhere but at $\pm z_{*}$, where it is $C^{1}$. Thus by the Itô-Tanaka formula we find:

$$
\begin{aligned}
e^{-2 t} W\left(Z_{t}\right)= & W\left(Z_{0}\right)+\int_{0}^{t} e^{-2 s}\left(\mathbb{L}_{Z} W\left(Z_{s}\right)-2 W\left(Z_{s}\right)\right) d s \\
& +\sqrt{2} \int_{0}^{t} e^{-2 s} W^{\prime}\left(Z_{s}\right) d \beta_{s} .
\end{aligned}
$$


Hence by (2.22) and the fact that $\mathbb{L}_{Z} W(z)-2 W(z)=0>-F(|z|)$ for $z \notin\left[-z_{*}, z_{*}\right]$, upon extending $W^{\prime \prime}$ to $\pm z_{*}$ as we please and using that the Lebesgue measure of those $t>0$ for which $Z_{t}= \pm z_{*}$ is zero, we get:

$$
e^{-2 t} W\left(Z_{t}\right) \geqslant W\left(Z_{0}\right)-\int_{0}^{t} e^{-2 s} F\left(\left|Z_{s}\right|\right) d s+M_{t},
$$

where $M=\left(M_{t}\right)_{t \geqslant 0}$ is a continuous local martingale given by

$$
M_{t}=\sqrt{2} \int_{0}^{t} e^{-2 s} W^{\prime}\left(Z_{s}\right) d \beta_{s}
$$

Using further that $W(z) \leqslant 0$ for all $z$, a simple application of the optional sampling theorem in the stopped version of (2.27) under $\mathbf{P}_{z}$ shows that $W_{*}(z) \geqslant W(z)$ for all $z$. To prove equality one may note that the passage from (2.26) to (2.27) also yields:

$$
0=W\left(Z_{0}\right)-\int_{0}^{\sigma_{*}} e^{-2 s} F\left(\left|Z_{s}\right|\right) d s+M_{\sigma *}
$$

upon using (2.19) and (2.20). Since clearly $\mathbf{E}_{z} \sigma_{*}<\infty$ and thus $\mathbf{E}_{z} \sqrt{\sigma_{*}}<$ $\infty$ as well, and $z \mapsto W^{\prime}(z)$ is bounded on $\left[-z_{*}, z_{*}\right]$, we can again apply the optional sampling theorem and conclude that $\mathbf{E}_{z} M_{\sigma_{*}}=0$. Taking the expectation under $\mathbf{P}_{z}$ on both sides in (2.3) enables one therefore to conclude $W_{*}(z)=W(z)$ for all $z$, and the proof of the claim is complete.

From (2.15) $-(2.17)$ and $(2.25)$ we find that $V_{*}=2 W_{*}(0)+1=2\left(\Phi\left(z_{*}\right)-\right.$ 1) $+1=2 \Phi\left(z_{*}\right)-1$. This establishes (2.2). Transforming $\sigma_{*}$ from (2.18) back to the initial problem via the equivalence of (2.9), (2.10) and (2.15), we see that $\tau_{*}$ from (2.4) is optimal. The proof is complete.

$\mathrm{R}$ e $\mathrm{m}$ a $\mathrm{rk} 1$. Recalling that $S-B \stackrel{\text { law }}{=}|B|$ we see that $\tau_{*}$ is identically distributed as the stopping time $\tilde{\tau}=\inf \left\{t>0:\left|B_{t}\right|=z_{*} \sqrt{1-t}\right\}$. This implies

$$
\mathbf{E} \tau_{*}=\mathbf{E} \tilde{\tau}=\mathbf{E}\left|B_{\tilde{\tau}}\right|^{2}=z_{*}^{2} \mathbf{E}(1-\tilde{\tau})=z_{*}^{2}\left(1-\mathbf{E} \tau_{*}\right),
$$

and hence we obtain

$$
\mathbf{E} \tau_{*}=\frac{z_{*}^{2}}{1+z_{*}^{2}}=0.55 \ldots
$$

Moreover, using that $\left(B_{t}^{4}-6 t B_{t}^{2}+3 t^{2}\right)_{t \geqslant 0}$ is a martingale, similar arguments show that

$$
\mathbf{E} \tau_{*}^{2}=\frac{z_{*}^{6}+5 z_{*}^{4}}{\left(1+z_{*}^{2}\right)\left(3+6 z_{*}^{2}+z_{*}^{4}\right)}=0.36 \ldots
$$

From (2.29) and (2.30) we find

$$
\mathrm{D} \tau_{*}=\frac{2 z_{*}^{4}}{\left(1+z_{*}^{2}\right)^{2}\left(3+6 z_{*}^{2}+z_{*}^{4}\right)}=0.05 \ldots
$$


$\mathrm{R}$ e $\mathrm{m} \mathrm{a} \mathrm{r}$ 2. For the sake of comparison with (2.2) and (2.29) it is interesting to note that

$$
V_{0}=\inf _{0 \leqslant t \leqslant 1} \mathbf{E}\left(B_{t}-\max _{0 \leqslant s \leqslant 1} B_{s}\right)^{2}=\frac{1}{\pi}+\frac{1}{2}=0.81 \ldots
$$

with the infimum being attained at $t=\frac{1}{2}$. For this, recall from $(2.8)$ and (2.6) that

$$
\mathbf{E}\left(B_{t}-S_{1}\right)^{2}=\mathbf{E} \int_{0}^{t} F\left(\frac{S_{s}-B_{s}}{\sqrt{1-s}}\right) d s+1
$$

where $F(x)=4 \Phi(x)-3$. Using further that $S-B \stackrel{\text { law }}{=}|B|$, elementary calculations show

$$
\begin{aligned}
\mathbf{E}\left(B_{t}-S_{1}\right)^{2}= & 4 \int_{0}^{t} \mathbf{E}\left(\Phi\left(\frac{\left|B_{s}\right|}{\sqrt{1-s}}\right)\right) d s-3 t+1 \\
= & 4 \int_{0}^{t}\left(1-\frac{1}{\pi} \operatorname{arctg} \sqrt{\frac{1-s}{s}}\right) d s-3 t+1 \\
= & -\frac{4}{\pi}\left(t \operatorname{arctg} \sqrt{\frac{1-t}{t}}+\frac{1}{2} \operatorname{arctg} \sqrt{\frac{t}{1-t}}-\frac{1}{2} \sqrt{t(1-t)}\right) \\
& +t+1 .
\end{aligned}
$$

Hence (2.32) is easily verified by standard means.

$\mathrm{R}$ e m a r k 3. In view of the fact that $\sigma_{*}$ from (2.18) with $z_{*}=$ $1.12 \ldots$ from (2.3) is optimal in the problem (2.17), it is interesting to observe that the unique solution to the equation $F(\widehat{z})=0$ is given by $\widehat{z}=0.67 \ldots$ Noting moreover that the map $z \mapsto F(z)$ is increasing on $[0, \infty)$ and satisfies $F(0)=-1$, we see that $F(z)<0$ for all $z \in[0, \widehat{z})$ and $F(z)>0$ for all $z>\widehat{z}$. The size of the gap between $\widehat{z}$ and $z_{*}$ quantifies the tendency of the process $|Z|$ to return back to the «favourable» region $[0, \widehat{z})$ where clearly it is never optimal to stop.

$\mathrm{R}$ e $\mathrm{m}$ a $\mathrm{r} \mathbf{k}$ 4. The case of a general time interval $[0, T]$ easily reduces to the case of a unit time interval treated above by using the scaling property of Brownian motion implying

$$
\inf _{0 \leqslant \tau \leqslant T} \mathbf{E}\left(B_{\tau}-\max _{0 \leqslant t \leqslant T} B_{t}\right)^{2}=T \inf _{0 \leqslant \tau \leqslant 1} \mathbf{E}\left(B_{\tau}-\max _{0 \leqslant t \leqslant 1} B_{t}\right)^{2}
$$

which further equals to $T\left(2 \Phi\left(z_{*}\right)-1\right)$ by $(2.2)$. Moreover, the same argument shows that the optimal stopping time in (2.32) is given by

$$
\tau_{*}=\inf \left\{0 \leqslant t \leqslant T \mid S_{t}-B_{t} \geqslant z_{*} \sqrt{T-t}\right\}
$$

where $z_{*}$ is the same as in Theorem 1 . 
$R$ e $m a r k$. The maximum functional in the argument above can be replaced by other functionals. The integral functional is an example which turns out to have a trivial solution.

Setting $I_{1}=\int_{0}^{1} B_{t} d t$ we find by Itô's formula that the following analogue of $(2.5)$ is valid:

$$
I_{1}=\int_{0}^{1}(1-t) d B_{t}
$$

Denoting $M_{t}=\int_{0}^{t}(1-s) d B_{s}$ it follows as in (2.8) that

$$
\mathbf{E}\left(B_{\tau}-I_{1}\right)^{2}=\mathbf{E}\left|B_{\tau}\right|^{2}-2 \mathbf{E}\left(B_{\tau} M_{1}\right)+\mathbf{E}\left|I_{1}\right|^{2}=\mathbf{E}\left(\tau^{2}-\tau\right)+\frac{1}{3}
$$

for all $\tau \in \mathscr{M}$. Hence we see that (cf. (2.40) below):

$$
\inf _{\tau \in \mathscr{M}} \mathbf{E}\left(B_{\tau}-I_{1}\right)^{2}=\frac{1}{12}=0.08 \ldots
$$

and that the infimum is attained at $\tau_{*} \equiv \frac{1}{2}$.

$\mathrm{R} \mathrm{e} \mathrm{m}$ a $\mathbf{r} \mathrm{k}$ 6. From the point of view of mathematical statistics, the «estimator» $B_{\tau}$ of $S_{1}$ is biased, since $\mathbf{E} B_{\tau}=0$ for all $0 \leqslant \tau \leqslant 1$ but $\mathbf{E} S_{1} \neq 0$. It is thus desirable to consider the values

$$
\begin{aligned}
& \widetilde{V}_{*}=\inf _{a \in \mathbf{R}, \tau \in \mathcal{M}} \mathbf{E}\left(a+B_{\tau}-S_{1}\right)^{2}, \\
& \widetilde{V}_{0}=\inf _{a \in \mathbf{R}, 0 \leqslant t \leqslant 1} \mathbf{E}\left(a+B_{t}-S_{1}\right)^{2}
\end{aligned}
$$

and compare them with the values from (1.1) and (2.29). However, by using that $\mathbf{E} B_{\tau}=0$ we also find at once that $a_{*}=\mathbf{E} S_{1}$ is optimal in (2.40) with $\tilde{V}_{*}=V_{*}-2 / \pi=0.09 \ldots$ and $\tilde{V}_{0}=V_{0}-2 / \pi=0.18 \ldots$

3. Stochastic integral representation of the maximum process. In this section we present a direct derivation of the stochastic integral representation (2.5) and (2.6) (cf. [3, p. 89-93] and [1, p. 363-369]). For the sake of comparison we shall deal with a standard Brownian motion with drift given by

$$
B_{t}^{\mu}=B_{t}+\mu t
$$

where $\mu$ is a real number. The maximum process $S^{\mu}$ associated with $B^{\mu}$ is given by

$$
S_{t}^{\mu}=\sup _{0 \leqslant s \leqslant t} B_{s}^{\mu}
$$

1. To derive the analogue of (2.5) and (2.6) in this case, we shall first note that stationary independent increments of $B^{\mu}$ imply

$$
\begin{aligned}
\mathbf{E}\left(S_{1}^{\mu} \mid \mathscr{F}_{t}^{B}\right) & =S_{t}^{\mu}+\mathbf{E}\left(\left(\sup _{t \leqslant s \leqslant 1} B_{s}^{\mu}-S_{t}^{\mu}\right)^{+} \mid \mathscr{F}_{t}^{B}\right) \\
& =S_{t}^{\mu}+\mathbf{E}\left(\left(\sup _{t \leqslant s \leqslant 1}\left(B_{s}^{\mu}-B_{t}^{\mu}\right)-\left(S_{t}^{\mu}-B_{t}^{\mu}\right)\right)^{+} \mid \mathscr{F}_{t}^{B}\right) \\
& =S_{t}^{\mu}+\left.\mathbf{E}\left(S_{1-t}^{\mu}-(z-x)\right)^{+}\right|_{z=S_{t}^{\mu}, x=B_{i}^{\mu}} .
\end{aligned}
$$


Using further the formula

$$
\mathbf{E}(X-c)^{+}=\int_{c}^{\infty} \mathbf{P}\{X>z\} d z
$$

we see that (3.3) reads as

$$
\mathbf{E}\left(S_{1}^{\mu} \mid \mathscr{F}_{t}^{B}\right)=S_{t}^{\mu}+\int_{S_{t}^{\mu}-B_{t}^{\mu}}^{\infty}\left(1-F_{1-t}^{\mu}(z)\right) d z \equiv f\left(t, B_{t}^{\mu}, S_{t}^{\mu}\right)
$$

where we use the following notation:

$$
F_{1-t}^{\mu}(z)=\mathbf{P}\left\{S_{1-t}^{\mu} \leqslant z\right\}
$$

and the map $f=f(t, x, s)$ is defined accordingly.

2. Applying Itô's formula to the right-hand side of (3.4), and using that the left-hand side defines a continuous martingale, we find upon setting $a_{\mu}=\mathbf{E} S_{1}^{\mu}$ that

$$
\begin{aligned}
\mathbf{E}\left(S_{1}^{\mu} \mid \mathscr{F}_{t}^{B}\right) & =a_{\mu}+\int_{0}^{t} \frac{\partial f}{\partial x}\left(s, B_{s}^{\mu}, S_{s}^{\mu}\right) d B_{s} \\
& =a_{\mu}+\int_{0}^{t}\left(1-F_{1-s}^{\mu}\left(S_{s}^{\mu}-B_{s}^{\mu}\right)\right) d B_{s}
\end{aligned}
$$

as a non-trivial continuous martingale cannot have paths of bounded variation. This reduces the initial problem to the problem of calculating (3.5).

3. The following explicit formula is well known (see e.g. $[2$, p. 368] or $[5$, p. $759-760])$ :

$$
F_{1-t}^{\mu}(z)=\Phi\left(\frac{z-\mu(1-t)}{\sqrt{1-t}}\right)-e^{2 \mu z} \Phi\left(\frac{-z-\mu(1-t)}{\sqrt{1-t}}\right) .
$$

Inserting this into (3.6) we obtain the representation

$$
S_{1}^{\mu}=a_{\mu}+\int_{0}^{1} H_{t}^{\mu} d B_{t}
$$

where the process $H^{\mu}$ is explicitly given by

$$
\begin{aligned}
H_{t}^{\mu}= & 1-\Phi\left(\frac{\left(S_{t}^{\mu}-B_{t}^{\mu}\right)-\mu(1-t)}{\sqrt{1-t}}\right) \\
& +e^{2 \mu\left(S_{t}^{\mu}-B_{t}^{\mu}\right)} \Phi\left(\frac{-\left(S_{t}^{\mu}-B_{t}^{\mu}\right)-\mu(1-t)}{\sqrt{1-t}}\right) .
\end{aligned}
$$

Setting $\mu=0$ in this expression, we recover (2.5) and (2.6).

4. Note that the argument above extends to a large class of processes with stationary independent increments (including Lévy processes) by 
reducing the initial problem to calculating the analogue of (3.5). In particular, the following «prediction» result deserves a special note. It is derived in exactly the same way as (3.4) above.

Let $X=\left(X_{t}\right)_{0 \leqslant t \leqslant T}$ be a process with stationary independent increments started at zero, and let us denote $S_{t}=\max _{0 \leqslant s \leqslant t} X$, for $0 \leqslant t \leqslant T$. If $\mathbf{E} S_{T}<\infty$ then the predictor $\mathbf{E}\left(S_{T} \mid \mathscr{F}_{t}^{X}\right)$ of $S_{T}$ based on the observations $\left\{X_{s} \mid 0 \leqslant s \leqslant t\right\}$ is given by the following formula:

$$
\mathbf{E}\left(S_{T} \mid \mathscr{F}_{t}^{X}\right)=S_{t}+\int_{S_{t}-X_{t}}^{\infty}\left(1-F_{T-t}(z)\right) d z
$$

where $F_{T-t}(z)=\mathbf{P}\left\{S_{T-t} \leqslant z\right\}$.

\section{REFERENCES}

1. Karatzas I., Shreve S. Methods of Mathematical Finance. Berlin: Springer-Verlag, 1998, $407 \mathrm{p}$.

2. Revuz D., Yor M. Continuous Martingales and Brownian Motion. Berlin: SpringerVerlag, 1994, 560 p.

3. Rogers L. C. G., Williams D. Diffusions, Markov Processes, and Martingales; V. 2: Itô's Calculus. Chichester: Wiley, 1987, 475 p.

4. Shiryayev A. N. Optimal Stopping Rules. New York: Springer-Verlag, 1978, 217 p.

5. Shiryayev A. N. Essentials of Stochastic Finance (Facts, Models, Theory). Singapore: World Scientific, 1999, 834 p. 